\title{
Aerodynamic Instabilities of Twin Circular Cylinders
}

\author{
M.Matsumoto, Assoc. Prof. \\ Dept. of Civil Eng., Kyoto Univ., Kyoto 606, Japan \\ N.Shiraishi, Prof. \\ Dept. of Civil Eng., Kyoto Univ., Kyoto 606, Japan \\ H. Shirato, Instructor \\ Dept. of Civil Eng., Kyoto Univ., Kyoto 606, Japan
}

\section{ABSTRACT}

The aerodynamic, or more generally fluid-dynamic, instability of the downstream circular cylinder in the wake of a similar cylinder in subcritical flow is the focal point of this paper. These phenomena are known to cause fatigue problems and impair the serviceability and safety of structures in various engineering fields. The aim of this study is to clarify the mechanism of this phenomena, as well as to find the conditions under which vibrations occur. A series of wind tunnel experiments and corresponding flow visualization in a water flume were conducted to permit discussion of the unsteady pressure properties, the aerodynamic unsteady force characteristics including its nonlinearity, and their relationship to the flow patterns.

The sudden decrease in pressure on the inward surface of the downstream cylinder when it approaches the upstream one, appears to play an important role in the vibration. Furthermore, an analogy between the unsteady aerodynamic force properties of the downstream cylinder and those of an isolated bluff body which undergoes both torsional and coupled flutter is postulated.

KEYWORDS: Aerodynamic vibration, Multiple structures, Wake-induced oscillation

\section{INTRODUCTION}

The aerodynamic problems of multiple structures in a flow field have frequently received the attention of engineers and other investigators for several decades. Multiple structures, which are placed in close proximity to each other in a flowing fluid, exhibit peculiar aerodynamic characteristics that are not seen on isolated structures. The resulting aerodynamic forces sometimes induce vibrations and cause noise which are undesirable for the structure's serviceability and safety. The generation of cracks in the supporting members of the pipes in heat exchangers due to vibration is one of the serious problems at present. In the civil engineering field, the evaluation of static wind loads on a structure, which are significantly influenced by the presence of another structure, remains a current problem in the design of structures. For example, the aerodynamic vibration of two separate, parallel bridges, and the vibration of twin cables in cable-stayed bridges have been discussed recently by a number of investigators (for example, Yokoyama et.al. [1985], Technical Report of JSCE and 
Honshu-Shikoku Bridge Authority [1985]).

Twin circular cylinders, whose aerodynamic characteristics were reviewed by Zdravkovich [1977a], are ranked as one of the most basic cross sectional shapes and show various aerodynamic vibration phenomena. These phenomena can be classified into two categories. One phenomenon, called "wake-induced flutter", occurs when the two cylinders are located so far apart that there is essentially no aerodynamic interference between the two cylinders, which is usually the case when $L / D>10$ approximately. (L: center-to-center distance between cylinders) The aerodynamic forces acting on the downstream cylinder can be described by the quasi-steady theory (for example, Simpson [1971], Price [1975], Cooper et.al. [1971]).

Aerodynamic vibrations, such as induced by flow interference, for example, "wake galloping" or "interference galloping" (Ruscheweyh [1983]), belong to different category. The spacing ratio $\mathrm{L} / \mathrm{D}$ is much smaller than 10 , with significant vibrations occurring in the region $L / D<5$. As the aerodynamic interference is dominant in this region, the induced aerodynamic forces are unsteady and cannot be well predicted by quasi-steady analysis (see, for example, Knisely and Kawagoe [1988]).

The authors' previous investigation on the aerodynamic instabilities of twin bluff bodies arranged in proximity (Shiraishi et.al. [1986]) has indicated that the generation mechanism for "interference galloping" for tandem circular cylinder was due to the unsteady effect of the "gap flow", as originally reported by Zdravkovich [1977b], and of the "outer accelerated flow". In the present paper, the aerodynamic vibration properties of the downstream circular cylinder will be discussed for large spacings through a series of wind tunnel experiments and flow visualization studies in a water flume. The aerodynamic forces, lift and drag, on the downstream cylinder were measured as well, in order to investigate their unsteadiness. In addition, an attempt to model the non-linear characteristics of these forces is made by applying a Duffing and van der Pol hybrid equation.

\section{EXPERIMENTAL APPARATUS}

All of the experiments were conducted in an Eiffel type wind tunnel with working area $2.5 \mathrm{~m}(W) \times 1.0 \mathrm{~m}(\mathrm{H}) \times 8.0 \mathrm{~m}(\mathrm{~L})$ and a water flume with an open channel of $1.0 \mathrm{~m}(W) \times 0.6 \mathrm{~m}(D)$ in the Department of Civil Engineering at Kyoto University. The smooth circular cylinder models were made of duraluminium with a diameter of $35 \mathrm{~mm}$, a span length of $930 \mathrm{~mm}$ and had end plates on both ends. The downstream one was equipped with 16 pressure taps along the circumference at the center of the span. The downstream model was supported by 8 coil springs so as to vibrate freely in the transverse and streamwise directions. Motions of the model in both streamwise $(X)$ and transverse $(Y)$ directions are detected by 2 single dimensional accelerometers (BAL-5G, Kyowa Electric Co. Ltd.) attached to the end plate.

The water flume was utilized to conduct flow visualization around twin circular cylinders undergoing vibration. The downstream cylinder is mounted vertically on an oscillating support which can control the motion of the model with an arbitrary frequency $(f)$, vibrating amplitudes $\left(X_{0}, Y_{0}\right)$ and also a phase difference between $X-$ and $Y-$ motion. The flow pattern is visualized by the hydrogen bubble method. In general, the upstream circular cylinder exhibits comparatively stable behavior (relative to that of the downstream cylinder) in the region of $v_{r}>1 / S_{t}\left(v_{r}\right.$ : reduced wind velocity, $\left.v_{r}=U / f D\right)$, except in the investigation by Bokaian and Geoola [1984] in which the aerodynamic instability of the upstream cylinder in an experimental study was reported and an analytical estimation was attempted. This aerodynamic instability was recorded when the distance between the two cylinders is rather small, L/D being about 1.5 which is closer than ones treated here. For this reason, the use of a fixed upstream cylinder in the present experiments is considered appropriate. 


\section{AERODYNAMIC VIBRATIONAL PROPERTIES OF DOWNSTREAM CYLINDER}

Figure 1 indicates the aerodynamic vibrational response of the downstream cylinder with 2 degrees of freedom, hereafter abbreviated d.o.f., in various arrangements. The region where significant response has been recorded should depend on the Scruton number of the cylinder. For the prototype cable, the Scruton number was evaluated as $O\left(10^{2}\right)$, which is approximately 10 times larger than that of this study. The region where the downstream cylinder vibrates in the Y- direction with 1 d.o.f. is also shown in the figure, and is approximately the same region as for 2 d.o.f. Therefore, the non-coupling term of the lift force is the predominant driving force, even for vibration with 2 d.o.f.

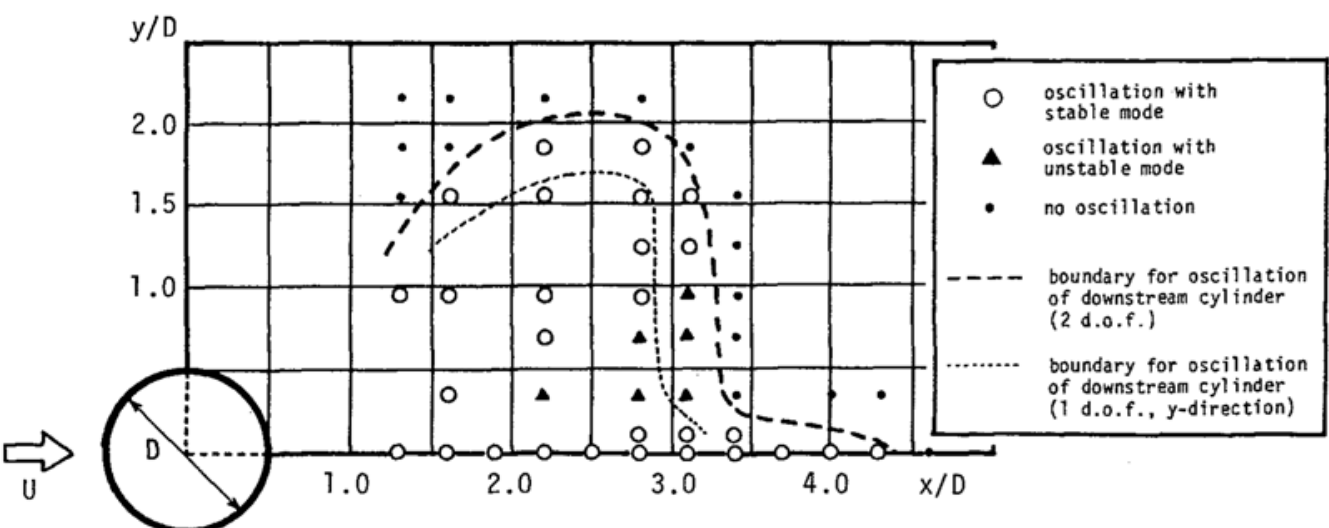

upstream Fig.1 Aerdodynamic vibrational response of the downstream cylinder cylinder with 2 d.o.f.

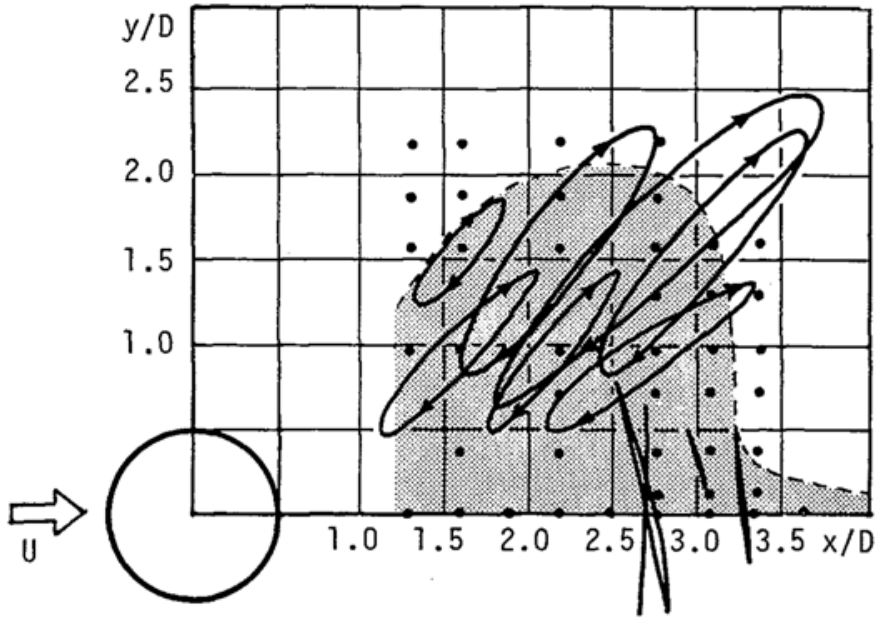

Fig.2 Vibrational mode of the downstream cylinder
Typical examples of the vibrational mode of the downstream cylinder in various arrangements are traced in Fig.2. The mode shapes are classified into 2 patterns, that is, the transverse dominant mode for the tandem arrangement, and the elliptical mode for the staggered arrangement. Between the regions where each of these two modes dominates is a region in which the vibrational modes are somewhat random and the amplitude is small. Yokoyama et.al.

[1985] reported the existence of the same stable region from the experiments of twin cable.

Figure 3 shows the unsteady surface pressure distribution on the downstream cylinder undergoing vibration $\left(V_{r}=124\right)$ in a staggered arrangement $(X / D, Y / D)=$ $(2.2,1.55)$. Corresponding steady surface pressures for a statically displaced downstream cylinder are also plotted in the figure. When the downstream cylinder is near its furthest position from the upstream one, no significant asymmetry can be recognized in the unsteady surface pressure distribution. 


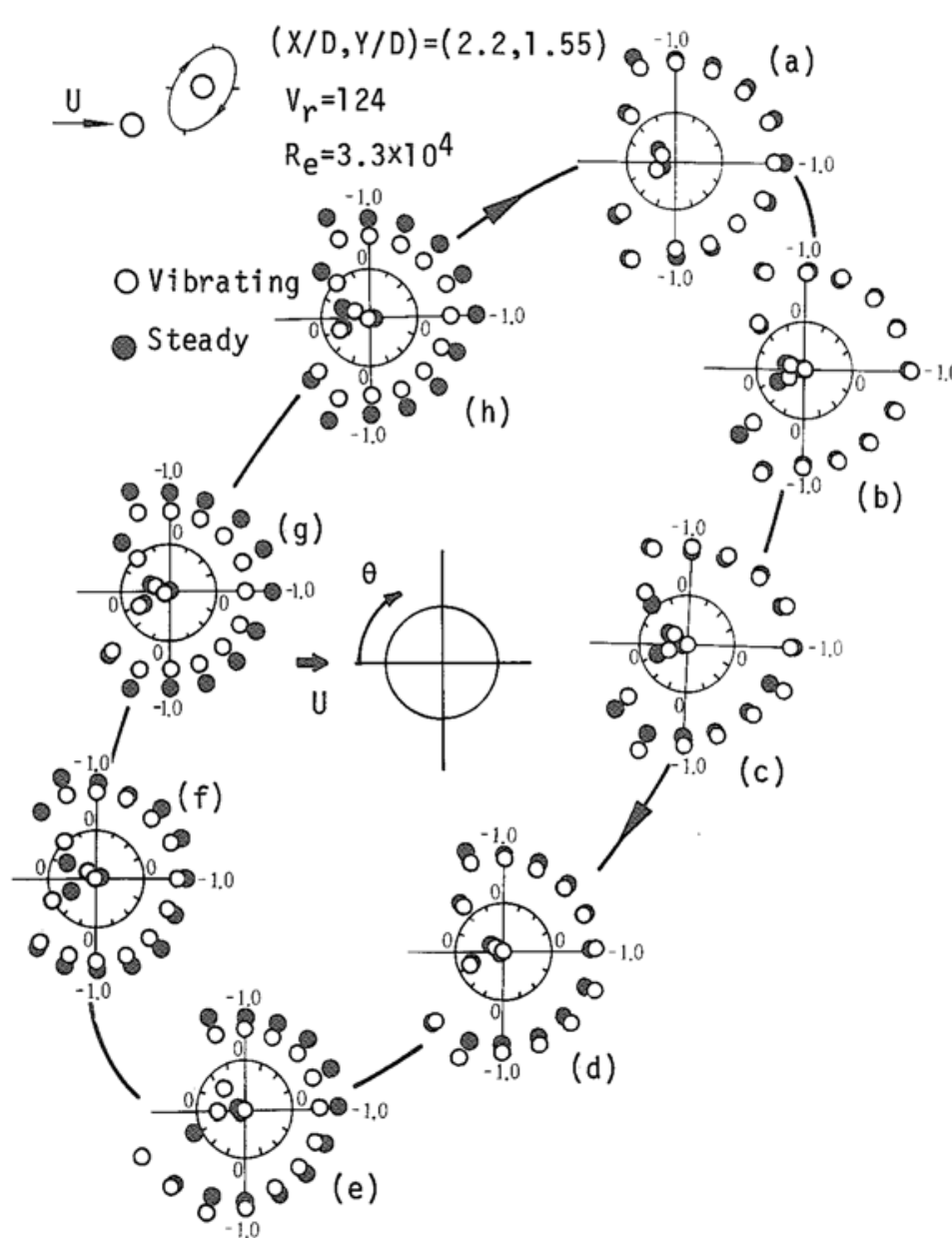

Fig. 3 Surface pressure distribution on the downstream cylinder
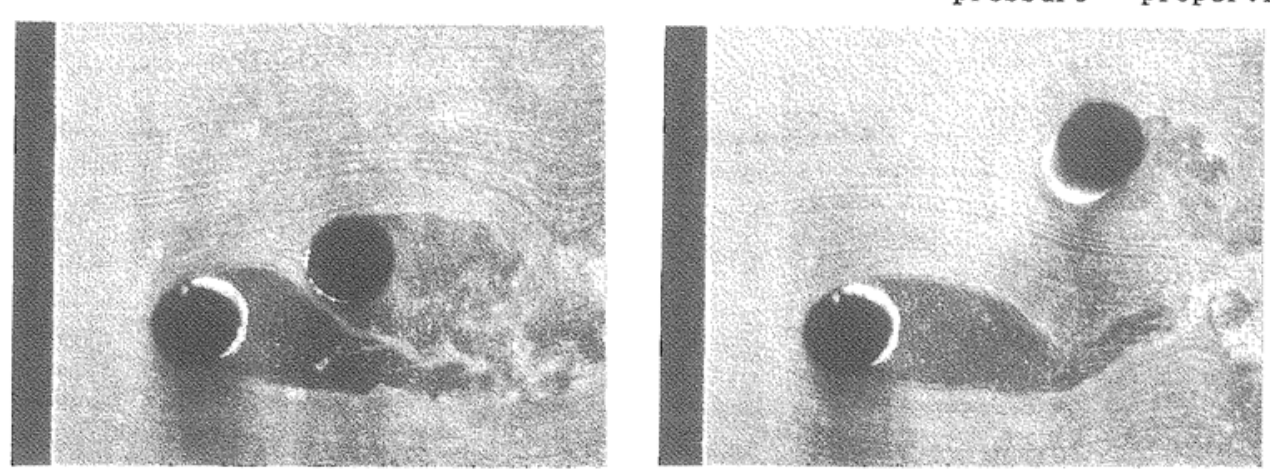

Fig. 4 Flow pattern around the cylinders $\left((X / D, Y / D)=(2.2,1.55), V_{r}=35.2, R e=1.49 \times 10^{4}\right)$

When the distance between the two cylinders approaches a minimum, both separation points on the downstream cylinder move to their extreme positions, that is, the outside separation point moves to its extreme downstream position, and the inside point to its extreme upstream position. In addition, the flow approaching the downstream cylinder tends to be directed toward the wake center, which also induces an inward directed lift force. These unsteady flow properties, as well 
as the unsteady pressure properties, are reinforced by the movement of the cylinder. The flow between the two cylinders, that is, the "gap flow", seems to be accelerated by the approach of the downstream cylinder. Consequently, the induced lift force during the half-period in which the downstream cylinder approaches to the upstream one is larger than that in the other half-period (see Fig.5).

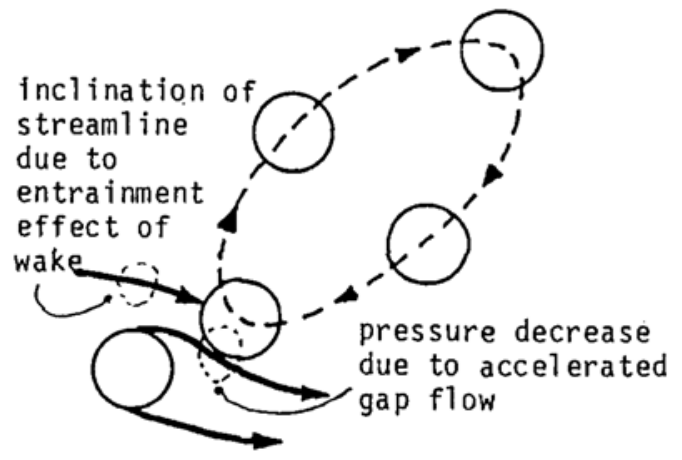

Fig.5 Vibration mechanism of the downstream cylinder

\section{UNSTEADY AERODYNAMIC FORCES ON DOWNSTREAM CYLINDER}

The unsteady lift and drag components on the downstream cylinder have been measured by the free vibration method. (Scanlan and Tomko, [1971]). The unsteady aerodynamic forces $F$ can be divided into two terms, those in-phase with the displacement $X$ and those in-phase with the velocity $\dot{X}$, where $X={ }^{T}(x, y)$.

$$
\mathrm{F}=(1 / 2) \rho \mathrm{U}^{2} \mathrm{D}\left\{\left[\begin{array}{cc}
\mathrm{k}^{2} \mathrm{~A}_{\mathrm{R}}^{*} & \mathrm{k}^{2} \mathrm{~B}_{\mathrm{R}}^{*} \\
\mathrm{k}^{2} \mathrm{C}_{\mathrm{R}}^{*} & \mathrm{k}^{2} \mathrm{D}_{\mathrm{R}}^{*}
\end{array}\right]\left\{\begin{array}{l}
\mathrm{x} / \mathrm{D} \\
\mathrm{y} / \mathrm{D}
\end{array}\right\}+\left[\begin{array}{cc}
\mathrm{kA}_{\mathrm{I}}^{*} & \mathrm{kB} \mathrm{B}^{*} \\
\mathrm{k} \mathrm{C}_{\mathrm{I}}^{*} & \mathrm{kD} \mathrm{D}^{*}
\end{array}\right]\left\{\begin{array}{l}
\dot{\mathrm{x}} / \mathrm{U} \\
\dot{\mathrm{y}} / \mathrm{U}
\end{array}\right\}\right.
$$

where, $\rho:$ air density, $U$ : approaching wind velocity,

D: cylinder diameter, $k$ : reduced frequency $(k=D \omega / U)$, $\omega:$ natural circular frequency,

$$
\mathrm{A}_{\mathrm{R}} * \mathrm{~B}_{\mathrm{R}}{ }^{*}, \mathrm{C}_{\mathrm{R}}{ }^{*}, \mathrm{D}_{\mathrm{R}}{ }^{*}, \mathrm{~A}_{\mathrm{I}}^{*}, \mathrm{~B}_{\mathrm{I}}{ }^{*}, \mathrm{C}_{\mathrm{I}}^{*}, \mathrm{D}_{\mathrm{I}}^{*}: \text { aerodynamic derivatives }
$$

Experimentally determined values for the aerodynamic derivatives are shown in Fig.6 for $(X / D, Y / D)=(2.2,1.55)$. Especially since the non-coupling component of the drag force, $A_{I}^{*}$ (which characterizes the aerodynamic damping force) takes a negative value, this non-coupling drag force component does not contribute significantly to the vibration. On the other hand, the non-coupling component of the lift force, $D_{I}^{*}$, takes on positive values at higher wind velocities, which can result in vibration. In addition, since $D_{R}{ }^{*}$ also takes on a positive value, it can be estimated that the non-coupling lift force, $D_{R}^{*} y+$ $\mathrm{D}_{\mathrm{I}}{ }^{*} \dot{\mathrm{y}}$, leads the $\mathrm{Y}$-displacement, and that the work done by this force component is positive. These unsteady lift force properties are the same as those for multiple pipes reported by Funakawa et.al. [1985] and Tanaka and Takahara [1981], both of which concluded that the mechanism of fluid-elastic vibration of multiple pipes is the phase lead of the lift force relative to the Y-displacement which is, in turn, due to the movement of the stagnation point in a direction opposite to that of body motion.

The sign of the energy transfer due to the coupling components of the unsteady aerodynamic forces, $\mathrm{B}_{\mathrm{R}}{ }^{*}(\mathrm{k}), \mathrm{B}_{\mathrm{I}}{ }^{*}(\mathrm{k}), \mathrm{C}_{\mathrm{R}}{ }^{*}(\mathrm{k}), \mathrm{C}_{\mathrm{I}}{ }^{*}(\mathrm{k})$, can be estimated by integrating over one cycle of motion. Work done by fluid on the vibration system can be expressed as :

$$
w_{D y}=\oint\left\{B_{R}^{*}(k) y+B_{I}^{*}(k) \dot{y}\right\} d x
$$



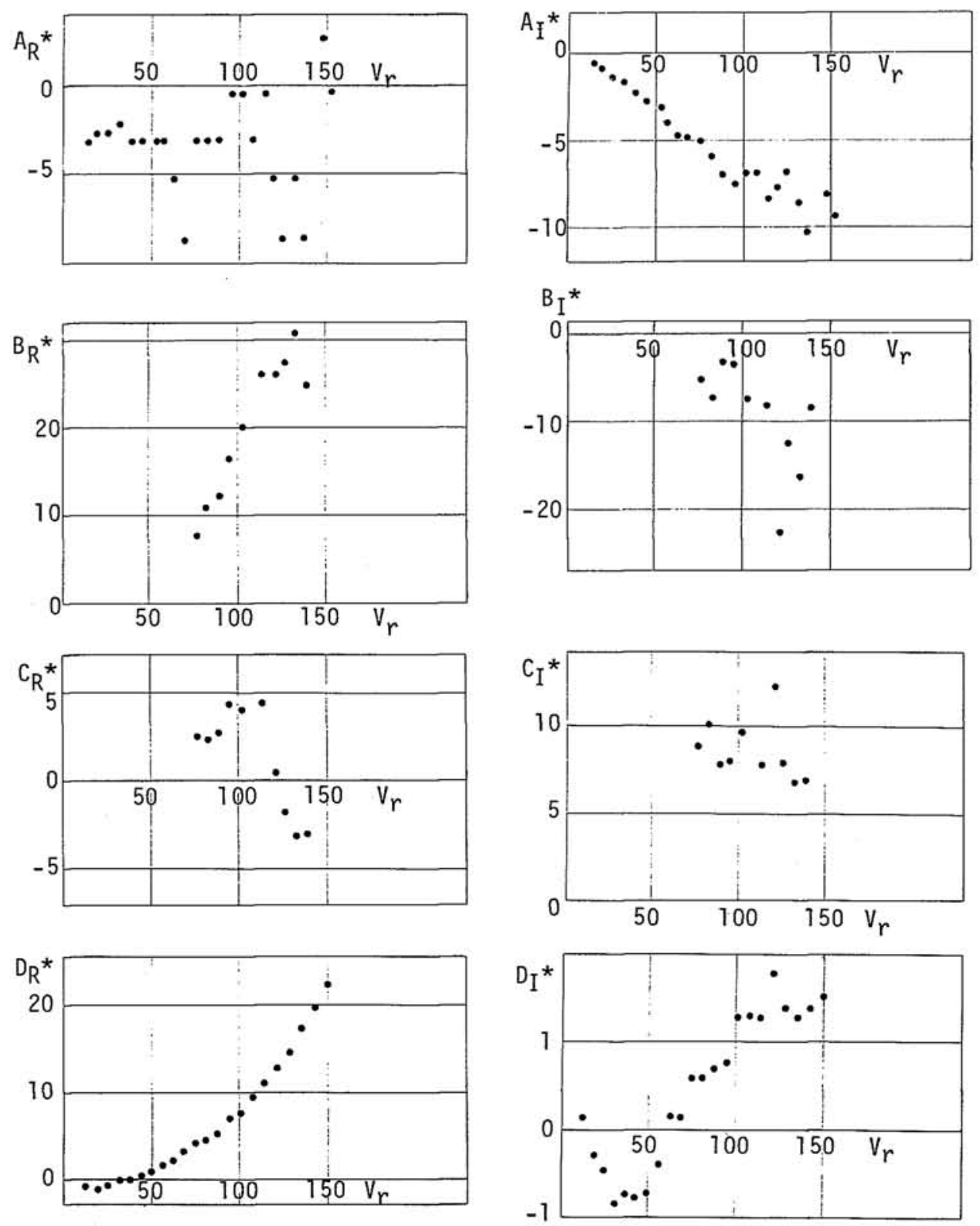

Fig.6 Aerodynamic derivatives

$$
\begin{aligned}
& =\mathrm{X}_{0} \mathrm{Y}_{0} \pi \omega\left(-\mathrm{B}_{\mathrm{R}}^{*}(\mathrm{k}) \sin \phi+\mathrm{B}_{\mathrm{I}}^{*}(\mathrm{k}) \omega \cos \phi\right) \\
W_{\mathrm{Lx}} & =\oint\left\{\mathrm{C}_{\mathrm{R}}{ }^{*}(\mathrm{k}) \mathrm{x}+\mathrm{C}_{\mathrm{I}}{ }^{*}(\mathrm{k}) \cdot \dot{\mathrm{x}}\right\} \mathrm{dy} \\
& =\mathrm{X}_{0} \mathrm{Y}_{0} \pi \omega\left(\mathrm{C}_{\mathrm{R}}{ }^{*}(\mathrm{k}) \sin \phi+\mathrm{C}_{\mathrm{I}}{ }^{*}(\mathrm{k}) \omega \cos \phi\right)
\end{aligned}
$$

where, $\quad x=x_{0} \sin \omega t, y=Y_{0} \sin (\omega t-\phi)$

By taking the signs of above aerodynamic derivatives into account, the phase difference, $\phi$, between $x$ and $y$ must obey the following inequalities. 
$\begin{array}{rlrrr}W_{\text {Dy }}>0 & \text { for } & \pi<\phi<(3 / 2) \pi \\ W_{\text {Lx }}>0 & \text { for } & -(1 / 2) \pi<\phi<0\end{array}$

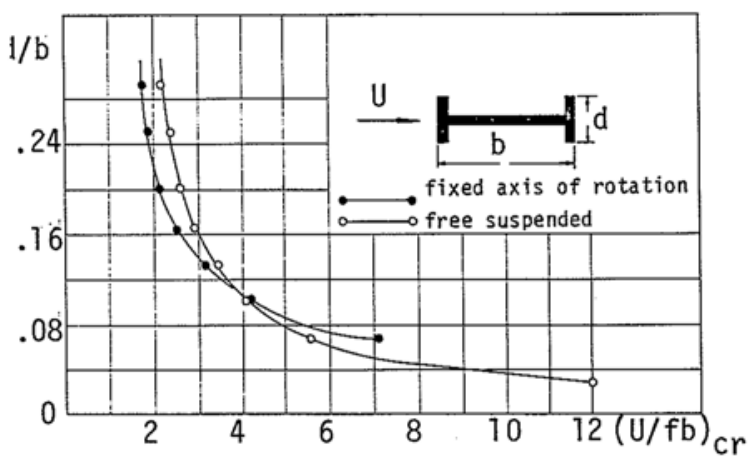

Fig.7 Dependence of flutter critical wind velocity on slenderness ratio $(d / b)$ and vibrational degree of freedom [Farquharson, 1949]
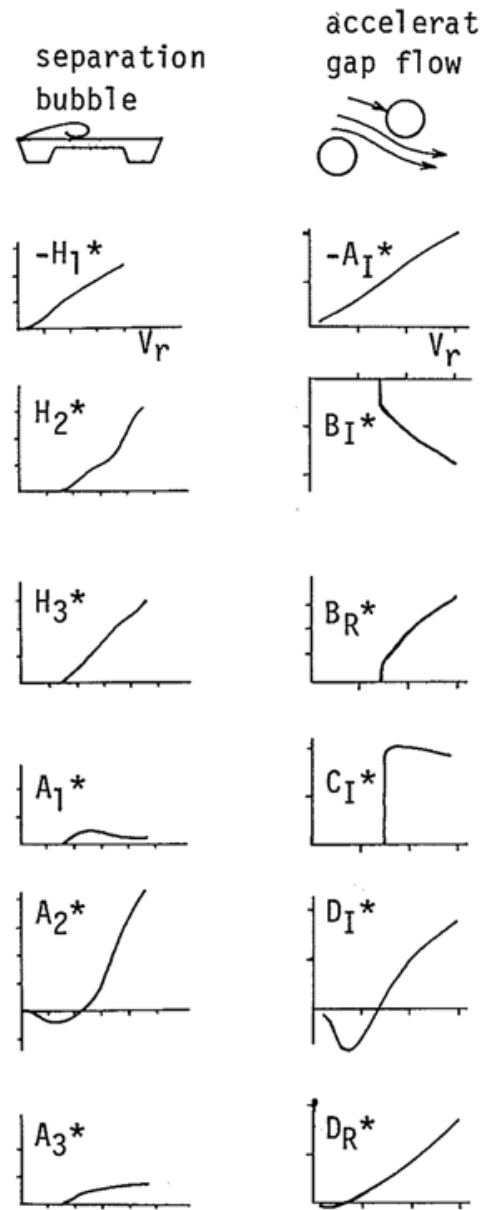

The experimentally determined phase difference $\phi$ exp was such that eq. (5) was satisfied. Thus, the predominant force providing energy to the vibrating system is the lift force component $\mathrm{L}_{\mathrm{X}}$.

As described above, the unsteady force component which plays the most important role in vibrations with elliptical orbits is the non-coupling lift forces, $\mathrm{L}_{\mathrm{y}}$. Moreover, the critical wind velocity tends to decrease when the system has 2 d.o.f., which is caused by the effect of coupling term of the lift force, $L_{X}$. An additional example of a structure which becomes more unstable for 2 d.o.f. than that for 1 d.o.f. is shown in Fig.7 [Farquharson, 1949], which indicates the dependence of critical wind velocity on the bluffness ratio, $d / b$, (d: depth; b: width of cross section) of an H-shaped cross section. Especially, sections in the range of $d / b$ between 0.067 and 0.1 exhibit aerodynamic instabilities in both torsional and coupled flutter, and the critical wind velocity for the 2 d.o.f. coupled flutter is lower than that for the 1 d.o.f. torsional mode. Figure 8 shows the aerodynamic derivatives for a 2-box girder cross section, which also exhibits aerodynamic instabilities for both flutter phenomena, compared with the derivatives for the downstream cylinder shown in Fig.6. Al though these two phenomena are different types of aerodynamic instability, there are many similarities in their unsteady force characteristics, if one compares the lift and moment components for the 2-box girder section to drag and lift for the downstream cylinder. Consequently, an analogy between the single cross section with 2 d.o.f. which exhibits both torsional and coupled flutter instabilities and for the downstream cylinder in staggered arrangement can be made.

Fig.8 Analogy of aerodynamic derivatives 


\section{NONLINEAR CHARACTERISTICS OF UNSTEADY FORCES}

The unsteady aerodynamic forces on the downstream cylinder which is placed in the wake of another identical circular cylinder have been expressed in terms of a component in phase with displacement and one in phase with the velocity in the last section. Since the amplitude of the downstream cylinder vibration is comparable to the cylinder diameter, D, the nonlinearity of these aerodynamic forces should be taken into account.

The nonlinear aerodynamic forces can be expressed as functions of vibrational amplitudes, $\xi_{0}$.

$$
F(t)=x_{\xi}\left(k, \xi_{0}\right) \cdot \xi+x_{\dot{\xi}}\left(k, \xi_{0}\right) \dot{\xi}
$$
where,
respect to $\mathrm{k}, \chi_{\xi_{0}}\left(\mathrm{k}, \xi_{0}\right), \chi_{\dot{\xi}}\left(\mathrm{k}, \xi_{0}\right):$ aerodynamic transfer function with

If one assumes that $F(t)$ can be expanded as an odd power series of $\xi$ and $\dot{\xi}$ as follows,

$$
F(t)=a_{1} \xi+a_{3} \xi^{3}+a_{5} \xi^{5}+\cdots+b_{1} \dot{\xi}+b_{3} \dot{\xi}^{3}+b_{5} \dot{\xi}^{5}+\cdots
$$

then substitutes $\xi=\xi_{0} \mathrm{e}^{\mathrm{i} \omega t}$ into Eq. (6), one obtains the following relation:

$$
\begin{aligned}
F(t)= & \left\{a_{1}+(3 / 4) a_{3} \xi_{0}^{2}+(5 / 8) a_{5} \xi_{0}^{4}+\cdots\right\} \xi \\
& +\left\{b_{1}+(3 / 4) b_{3}\left(\xi_{0} \omega\right)^{2}+(5 / 8) b_{5}\left(\xi_{0} \omega\right)^{4}+\cdots \cdots\right\} \dot{\xi}
\end{aligned}
$$

that is,

$$
\begin{aligned}
& \chi_{\dot{\xi}}\left(k, \xi_{0}\right)=a_{1}+(3 / 4) a_{3} \xi_{0}^{2}+(5 / 8) a_{5} \xi_{0}^{4}+\cdots \\
& \chi_{\dot{\xi}}\left(k, \xi_{0}\right)=b_{1}+(3 / 4) b_{3}\left(\xi_{0} \omega\right)^{2}+(5 / 8) b_{5}\left(\xi_{0} \omega\right)^{4}+\cdots
\end{aligned}
$$

$$
C_{L}(y, \dot{y})
$$

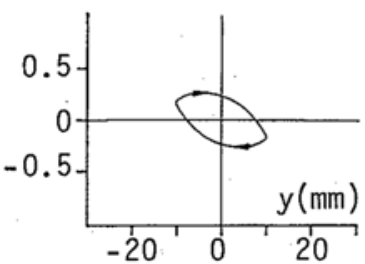

$c_{L}(y, \dot{y})$

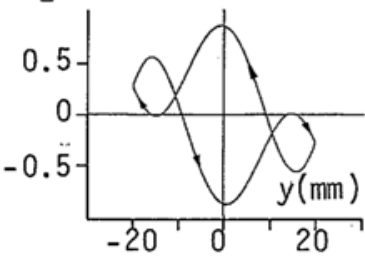

$$
C_{L}(y, \dot{y})
$$

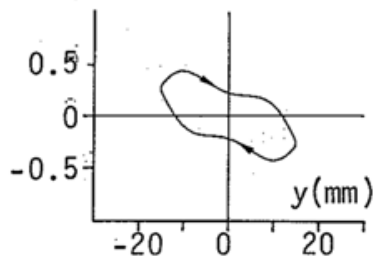

$$
C_{L}(y, \dot{y})
$$

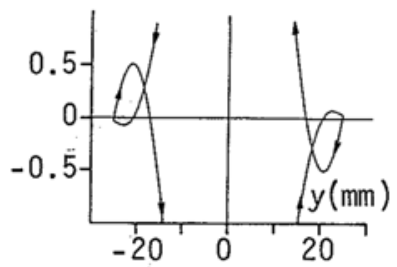

Fig.9 Nonlinearity of aerodynamic lift force with respect to vibrational ampl i tude $\left((X / D, Y / D)=(2.8,0), V_{r}=90.3\right)$

This method to determine $F(t)$ is none other than the method of Krylov \& Bogoliubov [1947] and Beecham-Tichener [Simpson, 1977] which have been applied to the conventional galloping or flutter of m.d.o.f. systems. Lissajous diagrams for $F(t)$ and displacement $y$ in case of $(X / D, Y / D)=(2.8,0)$ are shown in Fig.9. The shape of each diagram changes, especially, when $y$ is near 0 , depending on the vibrational amplitude $\mathrm{Y}_{0}$, which reflects a significant nonlinearity in $F(t)$. The effect of the "gap flow" and the "accelerated flow" govern $F(t)$ when $Y_{0}$ is relatively small. Since the so-called quasi-steady movement induced drag force $\mathrm{C}_{\mathrm{D}} \dot{\mathrm{y}} / \mathrm{U}$. tends to counteract these unsteady flow effects on $F(t)$ for larger $Y_{0}$, positive aerodynamic damping results for larger amplitudes. Because of the nonlinearity, the work 

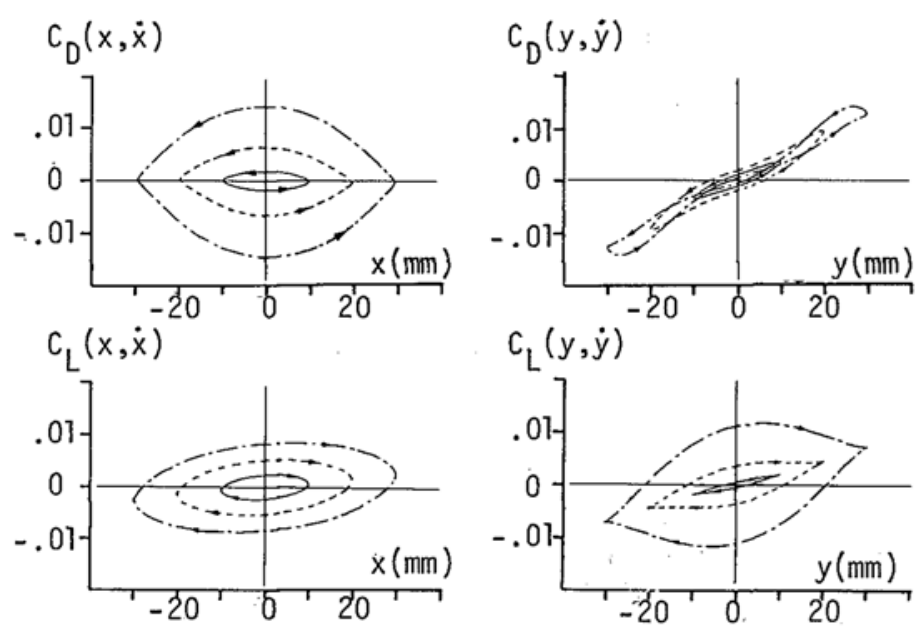

Fig.10 Nonlinearity of aerodynamic forces with respect to vibrational amplitude $\left((X / D, Y / D)=(2.2,1.55), V_{r}=80.2\right)$

done by $F(t)$ changes its sign from positive to negative with increasing vibrational amplitude, that is, $F(t)$ changes from an exciting force to a damping force with increasing ampli tude.

This treatment for $F(t)$ can be extended to 2 d.o.f. as indicated in Fig.10. While the drag force components $C_{D}(x$, $\dot{\mathrm{x}}), \quad \mathrm{C}_{\mathrm{D}}(\mathrm{y}, \dot{\mathrm{y}})$ indicate damping forces, the lift force components $\mathrm{C}_{\mathrm{L}}(\mathrm{x}, \dot{\mathrm{x}}), \mathrm{C}_{\mathrm{L}}(\mathrm{y}, \dot{\mathrm{y}})$ induce vibration, corresponding to the unsteady aerodynamic forces described. It should be noted that the nonlinearity is not dominant in these unsteady forces. Because this type of vibration occurs mainly outside of the wake, the variation of forces with displacement is not as significant as for the tandem arrangement.

\section{CONCLUSION}

(1) Concerning two circular cylinders arranged in proximity, the spatial region in. which significant vibration of the downstream cylinder possessing 2 d.o.f. can occur has been shown to be bounded by center to center spacings of between $2 \sim 4$, and the angle between the line of centers and approaching flow direction $45^{\circ}$.

The vibrational modes of the rigid spring mounted downstream cylinder can be classified into 2 types : a) an elliptical one with its axis inclined to the flow direction in staggered arrangements, b) transverse dominated one in tandem arrangements. The existence of the spatial region in which relatively small amplitude vibration with an unstable mode also confirmed. Since the downstream cylinder in staggered arrangements exhibits vibration even when it has 1 d.o.f. in $y$-direction, the important force component to generate vibration is the noncoupling lift force component.

(2) When the downstream cylinder undergoes vibration in the staggered arrangement, the unsteady pressure on the surface which faces toward both wake center line and upstream tends to decrease significantly when the downstream cylinder approaches the upstream one. This pressure change is due to the generation of the accelerated "gap flow" between the cylinders, and to the movement of the stagnation point toward the outside of wake center by the movement of cylinder and the entrainment effect of wake.

(3) The lift forces (both non-coupling and coupling terms) play an important role in the vibration with an elliptical orbit. An analogy exists between the drag and lift force components on the downstream cylinder and the lift force and pitching moment on the isolated cross section which exhibits both torsional and coupled flutter. Thus, the behavior of the "gap flow" and the "separation bubble" have similar effects on the aerodynamic vibration phenomenon.

(4) An attempt has been made to express the vibrational response properties of the downstream cylinder by expanding the aerodynamic unsteady forces $F(t)$ as power series in displacement $X$, and velocity $\dot{X}$, where $X={ }^{T}(x, y)$. By investigating Lissajous diagrams for the unsteady forces versus displacement of the downstream cylinder, the existence of an obvious nonlinearity in the unsteady 
lift force with respect to the vibrational amplitude for tandem cylinders was confirmed. For the elliptical orbit, the forces do not indicate so clear a nonlinearity, since the magnitudes of the fluctuating forces are relatively small compared with those in tandem arrangement.

\section{ACKNOWLEDGEMENTS}

The authors wish to thank Dr. C. Knisely for his technical comments and discussion of the manuscript and acknowledge the contribution of Mr. T.Memita, Mr. Y.Kim and Mr. M.Tsujii to a series of experiments and data analyses.

\section{REFERENCES}

Bokaian, A. and Geoola, F. 1984, "Proximity-Induced Galloping of Two Interfering Circular Cylinders", J. of Fluid Mech., vol.146, pp. 417-449.

Cooper, K.R. and Wardlaw, R.L. 1971, "Aerodynamic Instabilities in Wakes", Proc. of the Intn'l Conf. on Wind Effects on Build. and Struc., pp. 647-655.

Farquharson, F.B. 1949, "Aerodynamic Stability of Suspension Bridges with Special Reference to the Tacoma Narrows Bridge", Bull. of Univ. Washington Eng., Exp. Station, No.116, Part 1, pp.33.

Funakawa, M., Ishimatsu, T., Kumon, K. and Edakuni, K. 1985, "Mechanism of Fluiddynamic Instabilities on Tube Array", Proc. of Nat. Congress of JSME, No. 85-0053A, pp. 1-6.

JSCE and Technical Committee of Honshu Shikoku Bridge Authority 1985, Technical Report on Wind Resistance of Honshu-Shikoku Connecting Bridges, pp. 17-27.

Knisely, C.H. and Nakagawa, H. 1988, "Delay Time Model for Tandem Cylinder Vibration", Proc. of the 32nd JSCE Nat. Cong. on Hydraulics, pp. 559-564.

Krylov, N. and Bogoliubov, N. 1947, "Introduction to Non-Linear Mechanics", Analysis of Mathematical Studies, No.11.

Price, S.J. 1975, "Wake Induced Flutter of Power Transmission Conductors", J. Sound \& Vib., vol.38(1), pp. 125-147

Ruscheweyh, H.P. 1983, "Aeroelastic Interference Effects between Slender Structures", Proc. of the 6 th Intn'l Conf. on Wind Eng.

Scanlan, R.H. and Tomko, J.J. 1971, "Airfoil and Bridge Deck Flutter Derivatives", Trans. ASCE, EM6, pp. 1717-1737.

Shiraishi, N., Matsumoto, M. and Shirato, H. 1986, "On Aerodynamic Instabilities of Tandem Structures", J. of Wind Eng. and Ind. Aerod., vol.23, pp. 437-447.

Simpson, A. 1971, "Wake Induced Flutter of Circular Cylinders: Mechanical Aspects", The Aeron. Quart., vol.XXII, pp.101-118.

Simpson, A. 1977, "An Algorithm for Autonomous Non-Linear Dynamical Equations", The Aeron. Quart., pp.211-234.

Tanaka, H. and Takahara, S. 1981, "Fluid Elastic Vibration of Tube Array in Cross Flow", J. of Sound \& Vib., vol.77(1), pp. 19-37.

Yokoyama, K., Sato, H. and Kanzaki, K. 1985, "On Aerodynamic Properties of Twin Cables in Proximity Arrangements", Proc. of the 40 th Nat. Congress of JSCE, No. I-217.

Zdravkovich, M.M. 1977a, "Review of Flow Interference between Two Circular Cylinders in Various Arrangements", J. of Fluids Eng., ASME, pp. 618-633.

Zdravkovich, M.M. and Pridden, D.L. 1977b, "Interference between Two Circular Cylinders; Series of Unexpected Discontinuities", J. of Ind. Aerod., vol.2, pp. 255-270. 